\title{
Modeling community assembly on growing habitat "islands": a case study on trees and their vascular epiphyte communities
}

\author{
Lena Spruch $^{1} \cdot$ Jost Hellwig ${ }^{1,2} \cdot$ Gerhard Zotz $^{3,4} \cdot$ Bernd Blasius $^{1,5}$ (1)
}

Received: 6 December 2018 / Accepted: 21 March 2019

(C) Springer Nature B.V. 2019

\begin{abstract}
The number of available sites for establishment is a key determinant of species richness on habitat islands. While most theoretical studies assume habitat size or capacity to be constant, many natural habitats are characterized by dynamic growth in capacity over ecological timescales. A case in point is provided by trees that serve as habitat for vascular and non-vascular epiphytes. Here, we develop a modeling framework, based on neutral theory, to address the effects of habitat growth on community development, i.e., species richness and abundance. The model is parameterized to the situation of vascular epiphyte communities in tropical lowland forests and includes stochastic reproduction, death, and immigration events from a larger metacommunity. Using numerical simulations, we explore the proportion of growing sites occupied by individuals, the number of empty unoccupied sites, as well as changes in species abundances, species richness, colonization and extinction rates, and the dependence on the abundance in the metacommunity throughout the growth of the habitat. Our analysis suggests two characteristic phases of community development in a growing habitat: (i) an initial phase, characterized by a rapid buildup of empty sites, a slow increase in species abundance, and a fast increase in species richness, and (ii) a second phase, in which the number of empty sites reaches an equilibrium, species richness is accumulating very slowly, while the number of individuals increases unabatedly with habitat capacity.
\end{abstract}

Keywords Island biogeography $\cdot$ Neutral theory $\cdot$ Non-equilibrium $\cdot$ Biodiversity $\cdot$ Vascular epiphytes

\section{Introduction}

To identify the factors that determine community assembly and biodiversity patterns across space and time is one of the

Bernd Blasius

blasius@icbm.de

1 Institute for Chemistry and Biology of the Marine Environment (ICBM), Carl-von-Ossietzky University, Carl-von-Ossietzky Straße 9-11, 26129 Oldenburg, Germany

2 Environmental Hydrological Systems, Faculty of Environment and Natural Resources, University of Freiburg, Friedrichstraße 39, 79098 Freiburg, Germany

3 Institute for Biology and Environmental Sciences, Carl-von-Ossietzky University, Carl-von-Ossietzky Straße 9-11, 26129 Oldenburg, Germany

4 Smithsonian Tropical Research Institute, PO 0843-03092, Balboa, Ancon, Panama, Republic of Panama

5 Helmholtz Institute for Functional Marine Biodiversity, University of Oldenburg (HIFMB), Ammerländer Heerstraße 231,

26129 Oldenburg, Germany central challenges of modern ecology (Ricklefs and Schulter 1993). Since the publication of MacArthur and Wilson's theory of island biogeography (MacArthur and Wilson 1967), islands have been frequently used as model systems (Warren et al. 2015) in studies of community assembly and species richness (e.g., Kreft et al. 2007; Kier et al. 2009). Based on the implicit assumption of ecological neutrality among species, the theory of island biogeography predicts that the species richness of an island emerges as a dynamic equilibrium of extinction events and immigration from an external species pool. Thereby, apart from the degree of isolation, the size of an island holds a key role in determining species richness: Larger islands are able to support a larger number of species. Such species-area relationships generally describe the increase of species richness with habitat area; they are empirically well established and are commonly considered a fundamental pattern in ecology (Rosenzweig 1995; Lomolino 2000).

Hubbell (2001) has extended the theory of island biogeography to the unified neutral theory of biodiversity and biogeography, which describes ecological communities as open, continuously changing, non-equilibrium assemblages of 
species. In Hubbell's theory, species abundances are determined by stochastic immigration, speciation, and mortality events. Similar to the original theory of island biogeography, it assumes a constant habitat size and neutrality, but the neutrality assumption is extended from the species to the individual level. Additionally, Hubbell makes a zero-sum assumption i.e., the habitat is always saturated with individuals. Since its publication, Hubbell's theory has influenced the development of a variety of neutral models with different additions (Rosindell et al. 2011) and has been tested for empirical evidence, especially in tropical forests (Chave 2004). Both theories assume a constant habitat size, making them inapplicable to the investigation of the potential effect of changes in habitat size on community dynamics and biodiversity patterns.

Natural habitats of ecological communities are often dynamic; they are characterized not only by temporally changing environmental factors, but also by changes in size. Species assemblages that live in habitats changing in size exist in a variety of ecosystems and across diverse spatial scales, from communities on volcanic islands to epiphyte communities on trees to bacteria on marine organisms. Changes in habitat size should particularly affect communities that are physically dependent on other organisms ("foundation species"; Thomsen et al. 2018), as the host can considerably change in size throughout the development of the community and the life span of the species. Being a key factor for species richness, a continuously changing habitat size should have an impact on species richness patterns. However, theoretical approaches to study this effect are sparse. A few studies have focused on oceanic islands, where geological processes during island ontogeny influence biodiversity patterns with predictable temporal dynamics of species richness (Whittaker et al. 2007, 2008; Borregaard et al. 2015, 2017), but habitat growth that takes place over ecological timescales has not been addressed yet.

In this study, we propose a theoretical framework to address the effect of a growing habitat size on community development and temporal changes in species richness. As a direct application of this framework, we focus on vascular epiphytes in a tropical lowland forest. Epiphyte communities are an ideal and relevant subject for a case study: They are an essential element of tropical forests, accounting for almost $10 \%$ of all vascular plants globally (Zotz 2013) and for up to $50 \%$ of local plant diversity in montane rainforests (Kelly et al. 1994). The host trees that serve as habitat for epiphytes undergo significant changes in size during the establishment of the epiphyte community. The physical dependence on the host tree and the fact that biotic interactions, such as competition, seem to play a minor role in most epiphyte communities (Zotz 2016) are part of the reason why community dynamics of epiphytes are understood to a much lesser degree than those of other terrestrial plants. Theoretical approaches that exist, for example, for tropical trees (Condit et al. 2002) are difficult to apply to epiphytes. Species richness patterns of epiphytes are closely related to the morphological, architectural, and phenological characteristics of the respective host tree (Wagner et al. 2015). Host tree size, in particular, has been identified as an important factor influencing species richness and individual abundances of the epiphyte community: Species richness usually increases strongly with host tree size (Flores-Palacios and García-Franco 2006; Laube and Zotz 2006). As the tree ages, its characteristics change, which affects the development of the epiphyte community (Laube and Zotz 2007; Taylor and Burns 2015; Woods et al. 2015).

Several aspects of epiphyte community dynamics have been addressed in the context of the theory of island biogeography (Flores-Palacios and García-Franco 2006; MendietaLeiva and Zotz 2015), although the impact of tree ontogeny has typically been neglected. Studies of epiphyte communities in the tropics or temperate zones suggest that the epiphyte habitat is not saturated and that continuous growth of the community can be expected until the death of the host tree (Einzmann and Zotz 2017; Taylor and Burns 2015). Taylor and Burns (2015) adopted Darwin's geological theory of island ontogeny, which connects species richness to the stages of development of a volcanic island, to identify different stages of epiphyte community development throughout tree ontogeny.

In our approach to study community development in a dynamic habitat, we develop a stochastic model, based on the neutral theory established by Hubbell (2001), incorporating the continuous growth of the habitat and including stochastic reproduction, death, and immigration events from an external metacommunity. The model is adjusted to the parameters of vascular epiphyte communities in lowland forests in Panama. Using numerical simulations, we address the following questions: How does a growing habitat capacity affect the development of an epiphyte community? What is the proportion of colonization sites occupied by individuals compared with empty unoccupied sites that are continuously produced in such a growing habitat? How does the proportion of occupied and empty colonization sites change in time and how is this influenced by the rate of growth of the habitat capacity? What are the changes in species abundances, species richness, colonization and extinction rates, and the dependence on the abundance in the metacommunity throughout the growth of the habitat?

We are able to analyze the dynamics of empty and occupied colonization sites in terms of a simple ordinary differential equation (ODE) model. We find that, remarkably, the number of empty sites always reaches a stable equilibrium that is independent of habitat growth rate, although a temporary overshoot can be observed if the habitat is growing sufficiently fast. Combining the ODE model with full stochastic simulations, we obtain insights into the characteristic temporal patterns of the long-term development of a community in a growing habitat, which can be separated into two phases: (i) an 
initial phase of rapid buildup of empty sites because colonization does not keep pace with the development of new habitat, which is characterized by a fast increase in species richness and slow increase in total abundance, and (ii) a second phase, in which the number of empty sites reaches a steady state, species richness is increasing very little, while abundance increases unabatedly. By relating our model results to empirical data of epiphyte abundances and species richness from a lowland forest in Panama, we show that the model realistically captures the temporal development of epiphyte abundance and diversity during the colonization process on a growing tree.

\section{Methods}

\section{Model description}

We develop a spatially implicit individual-based model to simulate the neutral assembly of a community in a dynamic habitat (Fig. 1). The model closely follows neutral theory (Hubbell 2001), considering stochastic immigration of individuals from an external species pool and stochastic birth and death processes, but deviates from the usual zero-sum assumption. Rather than describing a fully saturated system of constant size, we assume that the habitat capacity $K(t)$, i.e., the total number of potential growing sites, is an increasing function of time $t$. These $K(t)$ sites are composed of sites occupied by individuals $N(t)$ and sites available for future settlement that are considered empty $E(t)=K(t)-N(t)$ (a site unit can be either empty or occupied by a single individual). When applied to simulate a community of vascular epiphytes growing on a single tree, the habitat capacity corresponds to the growing surface area of the tree that is considered potential substrate for epiphytes.

The model proceeds in discrete time steps of length $d t$. In each time step, first the habitat capacity is adjusted to its updated value $K(t)$. Thereby, the habitat capacity follows a deterministic (monotonously increasing) growth curve, which is independent of the settlement of organisms, and in general is a real, not necessarily integer, number. In this study, we usually assume a time-linear habitat growth

$K(t)=K_{0}+g t$

with growth rate $g$ or a constant habitat capacity $(g=0)$. We have confirmed that our results do not change qualitatively when other, non-linear growth functions are used (see Appendix 2 Fig. 10).

Next, the dynamics of the community are simulated by considering three stochastic processes (Fig. 1), reproduction $\left(a_{r}\right)$, death $\left(a_{d}\right)$, and immigration from a metacommunity $\left(a_{m}\right)$, with rates $a_{r}=r N(K-N)$

$a_{d}=d N$

$a_{m}=m(K-N)$

Following the neutrality assumption, these rates are identical for the individuals of all species in the community, but scale with the total number of individuals, $N$, for reproduction and death, and with the number of empty sites, $K-N$, for reproduction and immigration. Thus, population dynamics, i.e., reproduction, death, and immigration rates, are determined by the three positive parameters $r, d$, and $m$. To determine which of these population dynamic events takes place in a simulation time step, we follow a two-step procedure (Honerkamp 1993): We first decide if any event occurs based on the overall rate $u_{0}=a_{r}+a_{d}+a_{m}$. For this, we draw a uniformly distributed random number $r_{1} \in[0,1]$. If $r_{1}>u_{0} d t$, no population dynamic event takes place and the simulation proceeds to the next time step. Otherwise, if $r_{1} \leq u_{0} d t$, we generate another uniformly distributed random number $r_{2}$. Population dynamics event $\alpha$ (reproduction, death, or immigration) occurs if $y_{\alpha-1} \leq r_{2} \leq y_{\alpha}$, with $y_{\alpha}=\frac{1}{u_{0}} \sum_{i=1}^{\alpha} a_{i}$. Following this procedure, only one of the three processes can occur per time step. In the numerical simulation, we continually adjust the size of the time step $d t$ to the overall rate of the stochastic processes $u_{0}$ to ensure sufficiently small time steps, $u_{0} d t \ll 1$.

In the case of a reproduction event, an individual from the community is randomly chosen to reproduce, i.e., occupies one empty site. In the case of a death event, a random individual is removed from the community, increasing the number of empty sites by one. In the case of an immigration event, a new individual is randomly drawn from an external species pool, the metacommunity with a fixed relative species abundance distribution, and fills one empty site in the habitat. Thereby, the probability that the individual belongs to a certain species is proportional to the relative abundance of this species in the metacommunity. Since the habitat capacity $K(t)$ is a real number, it is theoretically possible that the number of empty sites obtains a value smaller than one, $0<E(t)<$ 1 , leaving no room for growth or settlement of a new individual. In this case (which did not occur in our simulations), we do not allow reproduction or immigration.

Iterating this scheme over many time steps, we are able to simulate the time dependence of the habitat capacity $K(t)$, the total number of individuals in the community $N(t)$, the number of empty sites $E(t)$, and the abundances $N_{i}(t)$ of each species. In addition, we are able to calculate the species richness $S(t)$, i.e., the number of species present in the habitat, the colonization rate, i.e., the rate of new species colonizing the habitat, as well as the extinction rate. 
Fig. 1 Conceptual diagram of the neutral community model in a growing habitat. Squares represent the sites that are available for colonization (but note that the model is not spatially explicit); the different colored shapes represent the different species. Starting from an initial state, in each time step, we iterate the following two processes: First, the habitat increases in capacity according to the continuous deterministic growth function (Eq. 1). Next, stochastic community dynamics can occur, depending on the rates of the individual processes (Eqs. 2-4): either a randomly chosen individual is reproducing (birth, reducing the number of empty sites by one, with rate $a_{r}$ ), or one random individual is removed from the community due to death (increasing the number of empty sites by one, with rate $a_{d}$ ), or one individual immigrates (reducing the number of empty sites by one, with rate $\left.a_{m}\right)$. In the case of an immigration event, the immigrating individual is randomly chosen from a metacommunity with a fixed relative species abundance distribution
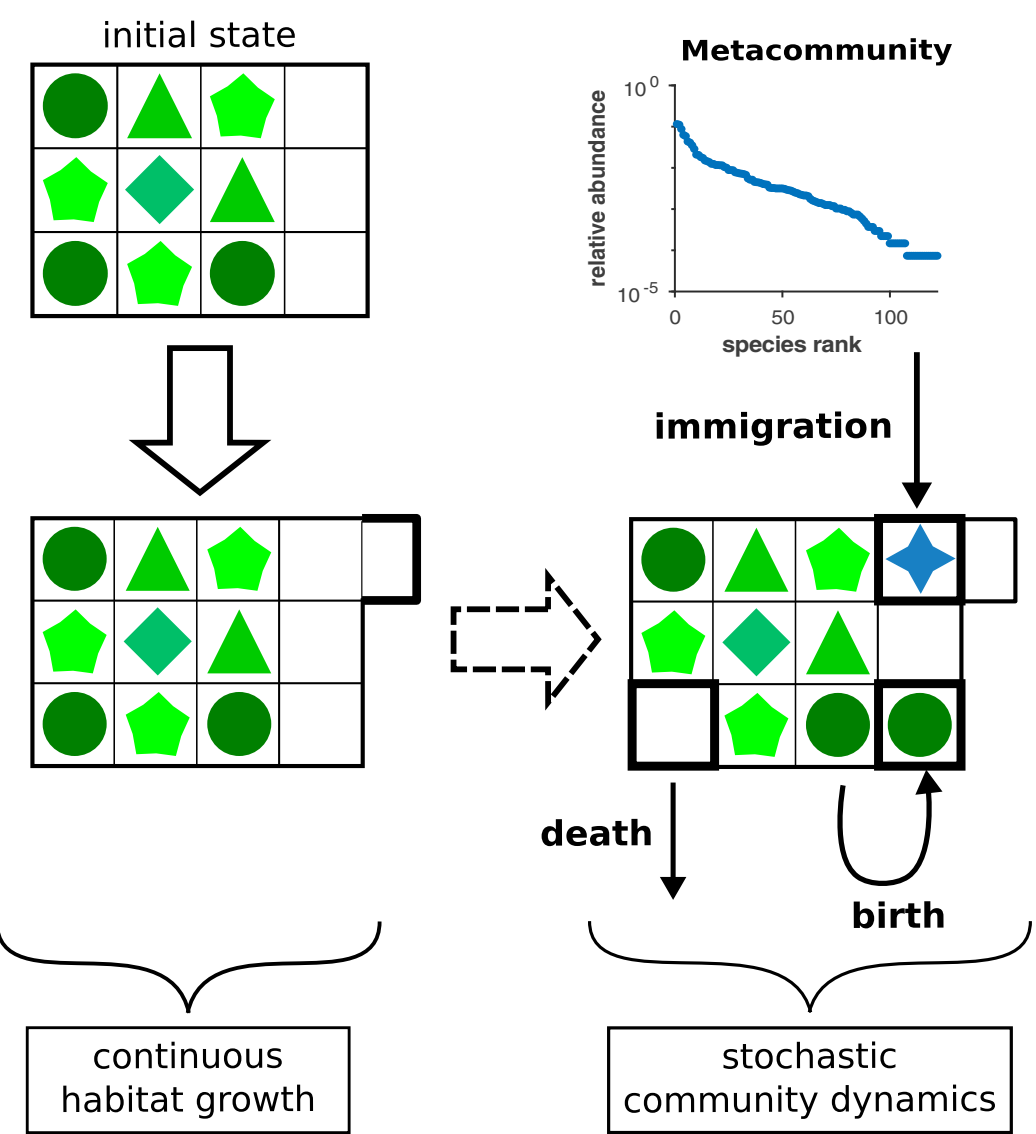

\section{Parameterization and data}

The model depends on the five parameters $r, d, m, g, K_{0}$ and the relative abundance distribution in the metacommunity. Parameterization is performed to describe the situation of vascular epiphyte communities in tropical lowland forests. We estimate the reproduction rate as $r=0.001$ year $^{-1}$, by averaging the data presented by Mondragón et al. (2015). Epiphyte death rate is set to $d=0.15$ year $^{-1}$, based on a study of the population dynamics of epiphytes in a Mexican humid montane forest by Hietz (1997). The immigration rate of individuals is set to $m=$ 0.03 year $^{-1}$, based on data on the colonization of empty host trees by epiphytes on 1200 Annona glabra trees by Laube and Zotz (2007). The tree growth rate is roughly estimated as $g=10$ year $^{-1}$ by calculating the increase in epiphyte individuals per tree per year between the census presented by Zotz and Schultz (2008) and the follow-up census (Mendieta-Leiva and Zotz, unpubl. data). For the model runs with a growing habitat, initial habitat capacity is always set to $K_{0}=1$. When the model is run with a constant habitat capacity, we set $K_{0}=1000$ to make the results comparable with the runs with a growing habitat over 100 years.

The relative species abundance distribution in the metacommunity is based on a census of vascular epiphytes in a lowland forest in Panama, comprising 13,574 individuals and 122 species (Zotz and Schultz 2008; see Appendix 1 Fig. 9). The data collected in these censuses includes epiphyte abundances and number of species per tree as well as the dbh (diameter at breast height) of each tree.

\section{Results}

\section{ODE model}

To gain first insights into the dynamics of a growing community, we derive a system of deterministic ODEs that captures the expected number of empty sites $E(t)$ and total number of individuals in the community $N(t)$. Assuming time-linear habitat growth with rate $g$, this yields

$\frac{d E}{d t}=d N-m E-r N E+g$

$\frac{d N}{d t}=-d N+m E+r N E$.

In contrast to the stochastic individual-based model described above, the ODE approach does not resolve the 
population dynamics of individual species $N_{i}(t)$, but can rather be regarded as a mean field model for the total number of individuals and empty sites in the habitat.

One can easily show that for a positive habitat growth rate, $g>0$, the model $(5,6)$ reaches a globally stable equilibrium value $\left(E^{*}\right)$ for the number of empty sites

$E^{*}=\frac{d}{r}$.

The equilibrium value is given as the ratio of per capita death and reproduction rates and is independent of the immigration rate, $m$, and the habitat growth rate, $g$ (as long as $g>0)$. In the case of a non-growing habitat $(g=0)$, the same equilibrium value $E^{*}=\frac{d}{r}$ is reached in the limit of a large initial habitat capacity $K_{0}=N(0)+E(0) \rightarrow \infty$. Note that Eq. 7 is not a true equilibrium of the $\operatorname{ODE}(5,6)$, because only the state variable $E(t)$ reaches a steady state. In contrast, the total number of individuals is not constant. After a transient period, it grows linearly in time according to the growth of the habitat $\dot{N}=g$.

To gain better insights into the model behavior, Fig. 2a, b investigates the model in the (E,N)-phase plane. Calculating the zero-growth isoclines $\dot{N}=0$ and $\dot{E}=0$ for the two state variables of the $\operatorname{ODE}$ model $(5,6)$ reveals two different patterns of how the equilibrium $E^{*}$ can be reached, depending on the relationship of the equilibrium value $E^{*}$ to the ratio $g / m$ of per capita growth and immigration rates. In the case of a slowgrowing habitat, $g / m<d / r$, the number of empty sites monotonically increases until it reaches the equilibrium value $d / r$ (Fig. 2a). In contrast, when the habitat is growing sufficiently fast, $g / m>d / r$, the number of empty sites temporarily exceeds the equilibrium level, giving rise to the curved trajectory in Fig. 2b. In both cases, the model reaches a state where the trajectory eventually moves straight up vertically in the phase plane, corresponding to the growing population (red curve in Fig. 2a, b). The overshooting for a rapidly growing habitat can be intuitively explained by the observation that for small population densities, the linear growth of the habitat can be faster than the exponential increase in population numbers. As a consequence, the habitat initially accumulates a large number of empty sites that are later occupied by the growing population. As shown in Appendix 2 Fig. 10, an equilibrium value for the number of empty sites is obtained also for other, e.g., exponentially or logarithmically, growing habitats.

This analysis revealed that the qualitative behavior of the ODE model depends only on two effective parameters: $d / r$ and $g / m$. The ratio of these two parameters influences not only how the number of empty sites reaches an equilibrium (Fig. $2 c)$, it has also a strong effect on the dynamics of the total number of individuals. This becomes most apparent when observing the fraction $\nu(t)=N(t) / K(t)$ of habitat sites occupied by individuals over time (Fig. 2d). Since the difference
$K(t)-N(t)$ approaches a finite value, the ratio $\nu(t)$ reaches a value of one for large times, $\lim _{t \rightarrow \infty} \nu(t) \rightarrow 1$. However, this limit can be approached in two different ways. When $g / m<d / r$ (slow-growing habitat, no overshooting), $\nu(t)$ is a concave, saturating function of time, while in the alternative case (fast-growing habitat, overshooting of empty sites), it resembles a sigmoid curve. As a consequence, the fraction of occupied sites in general will be smaller in a situation with overshooting compared with a case without overshooting. The difference between these two cases can be considerable. For the parameterization of Fig. 2 after a simulation time of about 40 years, $40 \%$ of the habitat is occupied by individuals in the case of $g / m<d / r$, while only $10 \%$ is occupied when $g / m>d / r$ (Fig. 2d). These patterns from the ODE model are confirmed by simulations with the full stochastic model. As shown in Fig. 3, the results of the ODE model coincide with the results of the stochastic model.

\section{Stochastic model}

Next, we investigate the full stochastic model. We start by comparing two scenarios: a habitat with a constant habitat capacity $(g=0)$ and a habitat with continuous and linear growth of $g=10$ sites/year. Using stochastic simulations, we determine for both habitat types the time dependence of the number of empty sites $E(t)$, the total number of individuals $N(t)$, the relative abundance for all species in the community $N_{i}(t)$, and the species richness $S(t)$ (Fig. 4). In the non-growing habitat with constant habitat capacity $\left(K_{0}=\right.$ 1000 ), the number of occupied and empty sites reach a steady state with $N^{*}=855 \pm 11$ and $E^{*}=145 \pm 11$ sites in less than ten simulated years (Fig. 4a). The dynamics of the abundances of individual species $N_{i}(t)$ show that the most common species of the metacommunity can establish quickly and permanently in the habitat while rarer species undergo large fluctuations in abundance and go extinct and reestablish more often (Fig. 4c). Species richness rises quickly until it reaches a stationary value of $S^{*}=36 \pm 3.6$, although the community exhibits strong fluctuations in individual model runs (Fig. 4e).

These results differ markedly from the scenario of a habitat with linear growth (Fig. 4, right column). In this simulation, we use a growth rate of $g=10$ sites per year and initialize the habitat with a size of $K_{0}=1$, so that a habitat size identical to that in the scenario of a non-growing habitat $\left(K_{0}=1000\right)$ is reached after 100 years. As shown in Fig. 4b, with the growing habitat, the total number of individuals in the community, $N(t)$, does not reach an equilibrium value. Instead, $N(t)$ is monotonously increasing, and after some initial transient, this increase is linear in time, following the growth of the habitat. At the same time, while both the habitat capacity and the number of individuals are increasing, the number of empty 

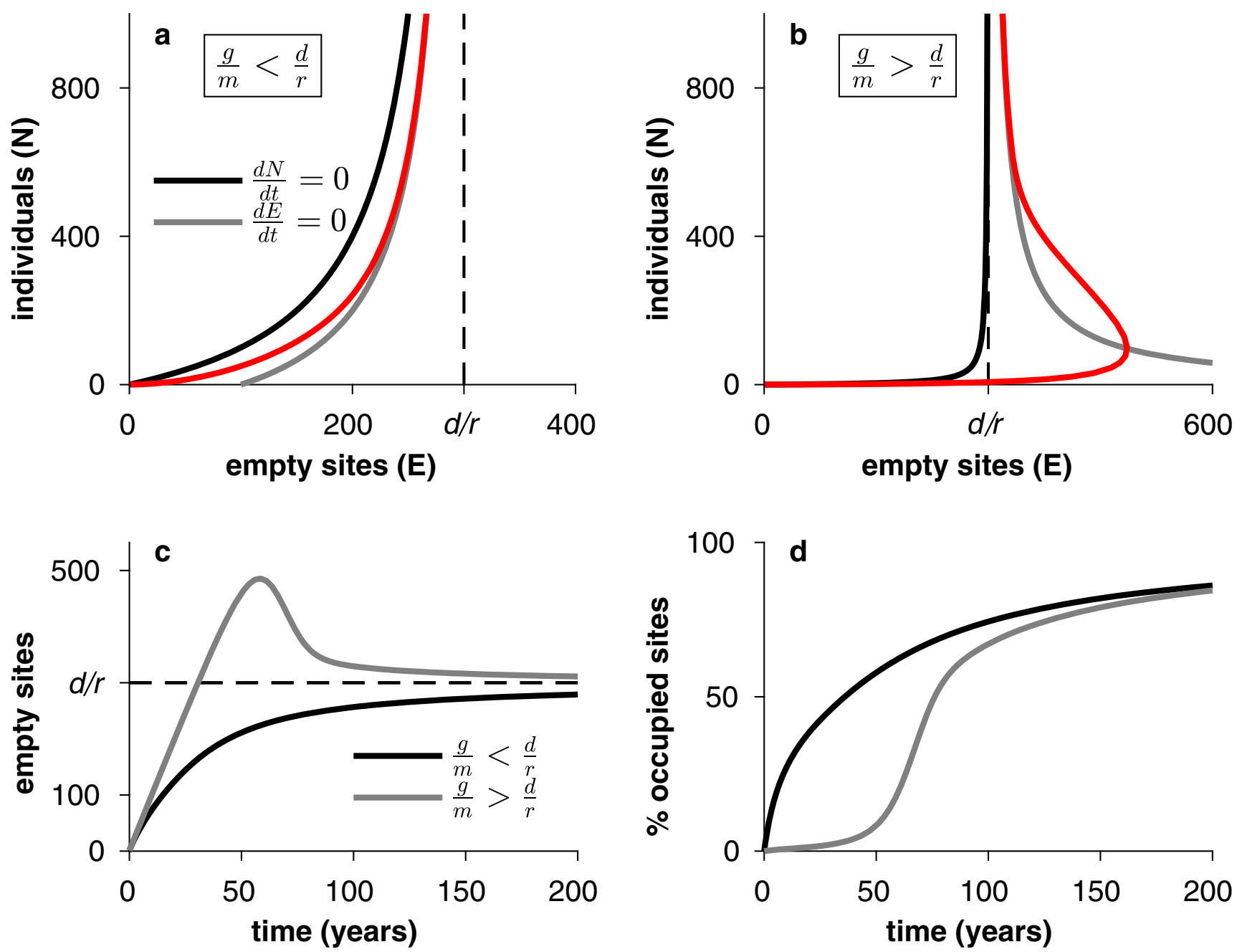

Fig. 2 The two characteristic patterns how the equilibrium of empty sites can be reached in a growing habitat. Shown are the results of the ODE model $(5,6)$ for the case of $g / m<d / r$ (no overshooting) and $g / m>d / r$ (overshooting, the number of empty sites temporarily exceeds its equilibrium value). a, b Phase portraits, showing the trajectory in the (E,N)-phase plane (red), as well as the zero growth isoclines $\dot{N}=0$

(black) and $\dot{E}=0$ (grey). c Time series of the number of empty sites $E(t)$ and $\mathbf{d}$ percental fraction of occupied sites, $N(t) / K(t)$, for a duration of 200 years; different colors distinguish the cases without overshooting (black) and with overshooting (grey). Parameter values are $r=$ 0.001 year $^{-1}, d=0.15$ year $^{-1}, g=10$ year $^{-1}$, and $m=0.1$ year $^{-1}$ (no overshooting) or $m=0.001$ year $^{-1}$ (overshooting)
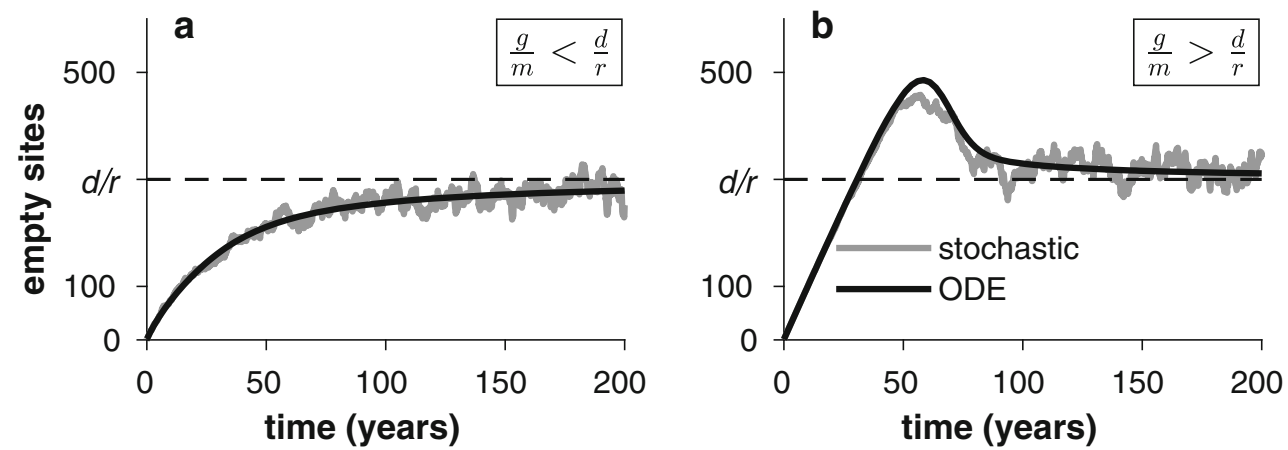

Fig. 3 Comparison of the results from the stochastic individual-based and the mean field model. The plots show the temporal development of the number of empty sites $E(t)$ simulated with the ODE model $(4,5)$ (black) and the stochastic model (grey) for the two scenarios: $g / m<d / r$ (a $m=$ 0.1 year $\left.^{-1}\right)$ and $g / m>d / r\left(\mathbf{b} m=0.001\right.$ year $\left.^{-1}\right)$. The two scenarios illustrate

the two patterns how the equilibrium number of empty sites $E^{*}=d / r$ (horizontal dashed line) can be reached (see also Fig. 2): either as a monotonic function (a) or with temporary overshooting (b). Parameter values as in Fig. 2 
sites $E(t)$ reaches an equilibrium value of $E^{*}=150$ sites, in accordance with Eq. 7 from the ODE model. This state is reached after a transient time of about 30 years, during which $E(t)$ undergoes a slight overshooting (as predicted by the ODE model). Following the increase of $N(t)$, the abundances of individual species $N_{i}(t)$ are, on average, constantly increasing as well (Fig. 4d). Similar to the scenario with a constant habitat capacity, the most common species of the metacommunity are able to establish first in the habitat and are present the longest. Despite the overall growth in the abundance of
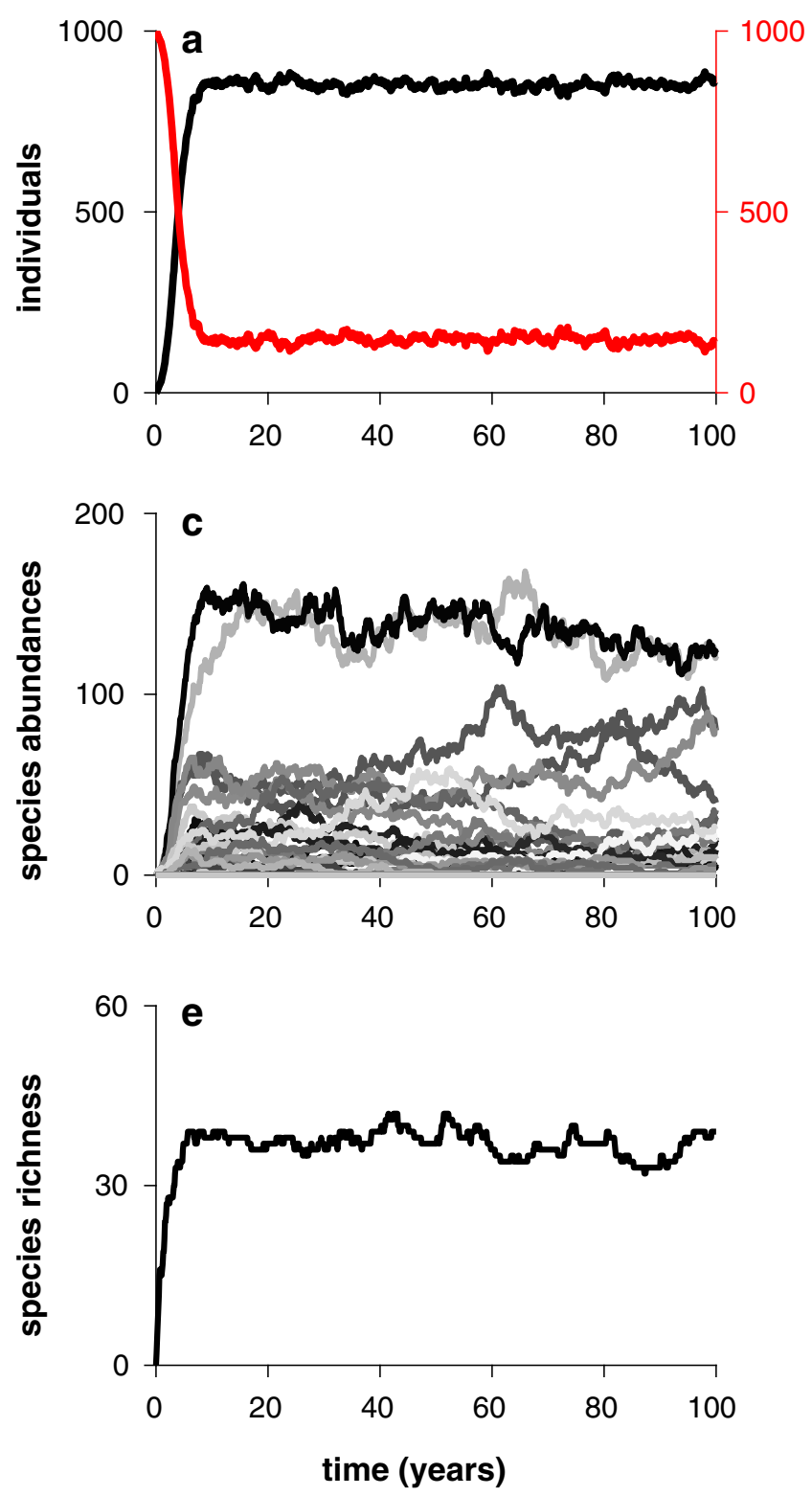

Fig. 4 Typical community dynamics in a static (left column) and a linearly growing (right column) habitat. In the static habitat $(g=0)$, the number of available growing sites is fixed to $K_{0}=1000$. The growing habitat is initialized with a size of $K_{0}=1$ and grows with a rate of $g=$ 10 sites/year, so that habitat sizes are identical at the end of the simulation at $t=100$ years. The remaining parameter values for both cases are $r=$ individual species, the rank abundance distribution remains rather constant. Species richness continues to increase gradually as more growing sites become available (Fig. 4f). With the parameter values of Fig. 4, after 100 years, species richness increases to a mean value of $S=36 \pm 3.6$. Thus, in both scenarios of static and growing habitats, the species richness after 100 years represents about $30 \%$ of the species in the metacommunity. Notice though that this value depends on model parameters and simulation time: In a growing habitat species, richness continues to increase with tree age and may
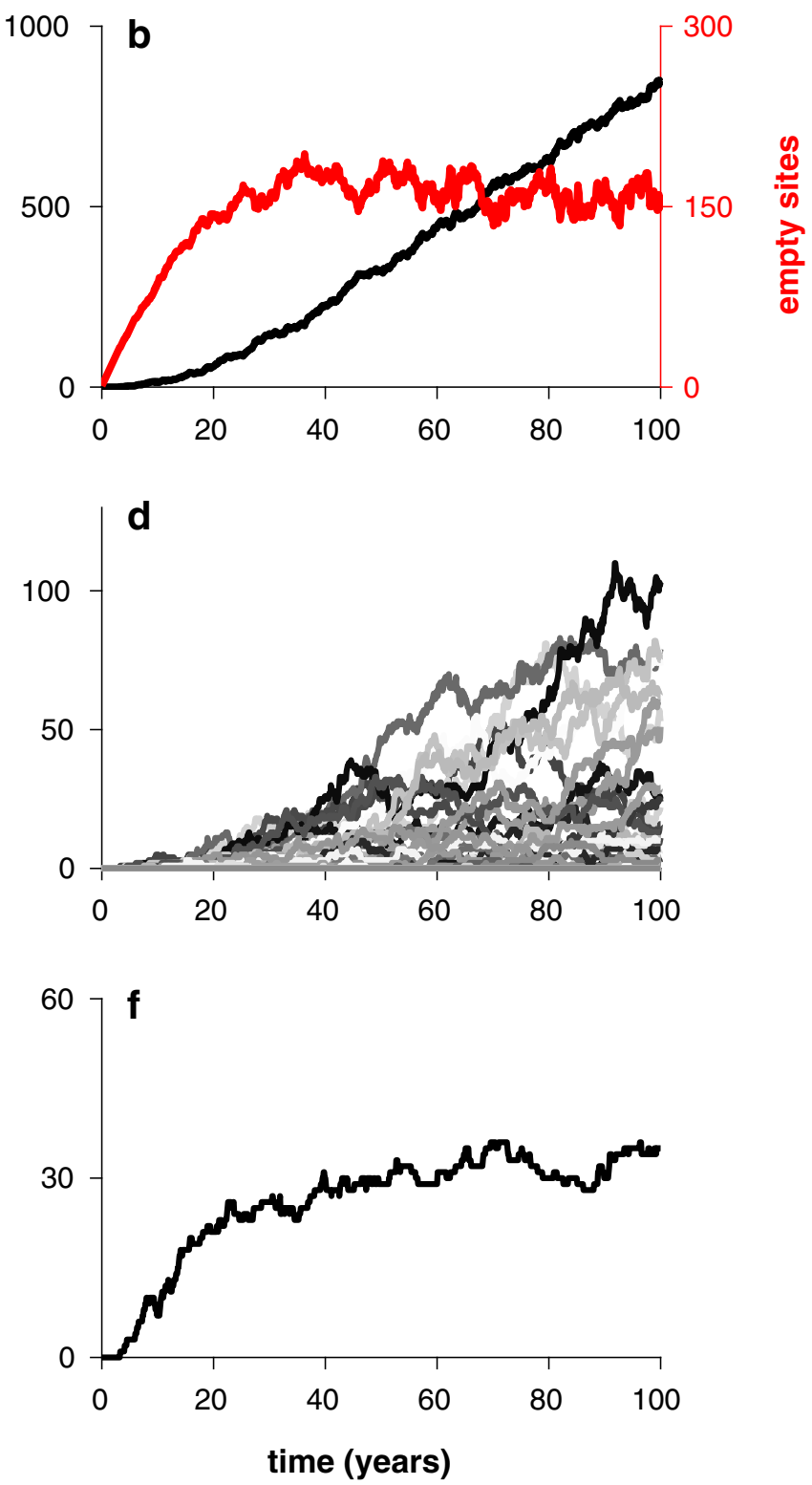

0.001 year $^{-1}, d=0.15$ year $^{-1}$, and $m=0.03$ year $^{-1}$ — corresponding for $g=10$ year $^{-1}$ to a situation with slight overshooting of empty sites. Shown are the results of the stochastic model as a function of time: $\mathbf{a}, \mathbf{b}$ total number of individuals $N(t)$ (black) and empty sites $E(t)$ (red) in the respective habitat, $\mathbf{c}, \mathbf{d}$ abundances of the individual species (different grey shadings), and e, f species richness $S(t)$ 
well reach $50 \%$ of the species in the metacommunity (see Fig. 8 below).

In Fig. 5, we systematically investigate the parameter dependence of community properties by averaging model outcomes over 1000 simulation runs of the stochastic model after a transient of 100 years. With respect to the equilibrium value of empty sites, these simulations confirm the analytic result, Eq. 7, from the ODE model for a growing habitat: The equilibrium value of $E^{*}$ is independent of the immigration rate $m$ (Fig. 5a), it is decreasing with increasing per capita reproduction rate $r$ (Fig. 5b), and it is linearly increasing with the per capita death rate $d$ (Fig. 5c). In the non-growing habitat, the parameter dependence of $E^{*}$ is very similar, but it always achieves a slightly smaller value than in the growing habitat (Fig. $5 \mathrm{a}-\mathrm{c}$ ). In contrast, the mean species richness $S$ in the static and dynamic habitats reaches similar values for varying parameter values after a simulation time of 100 years. As will be shown below, this is different for considerably longer simulation runs, because in the dynamic habit, species richness continues to increase over time. In general, species richness is proportional to immigration rate $m$ (Fig. 5d). It decays with the per capita reproduction rate $r$ (Fig. 5e) and is almost unaffected by the per capita death rate $d$ (Fig. 5f). As shown in Fig. 5d-f, even a 50\% increase in death rate does not significantly change the resulting species richness, while a similar increase in immigration and reproduction rates causes species richness to change by, respectively, $16 \%$ and $14 \%$.

Our simulations reveal a characteristic temporal pattern in the long-term development of a plant community in a growing habitat. This is demonstrated in Fig. 6 for the case of a fastgrowing habitat with an overshooting of empty sites $(g / m>d /$ $r$ ), showing the typical time course of an increasing number of individuals $N(t)$ and a temporary overshooting in the number of empty sites $E(t)$ (compare with Fig. 2). The figure also shows the time courses of colonization (here defined as the rate of successful colonization events of new species from the metacommunity into the habitat) and extinction rates, which exhibit an intermittent maximum similar to $E(t)$. The longterm development of species richness shows a fast increase in the beginning, followed by a considerable slow down after about 30 years, which coincides with the time at which the number of empty sites reaches its maximal value. Accordingly, the colonization rate has its peak very early, with a mean of two colonization events per year. With increasing species richness, the colonization rate is reduced because the probability that a new immigrating individual is already represented by an existing species in the habitat increases. The temporal pattern of the colonization rate is followed by the extinction rate, which shows a maximum of one event per
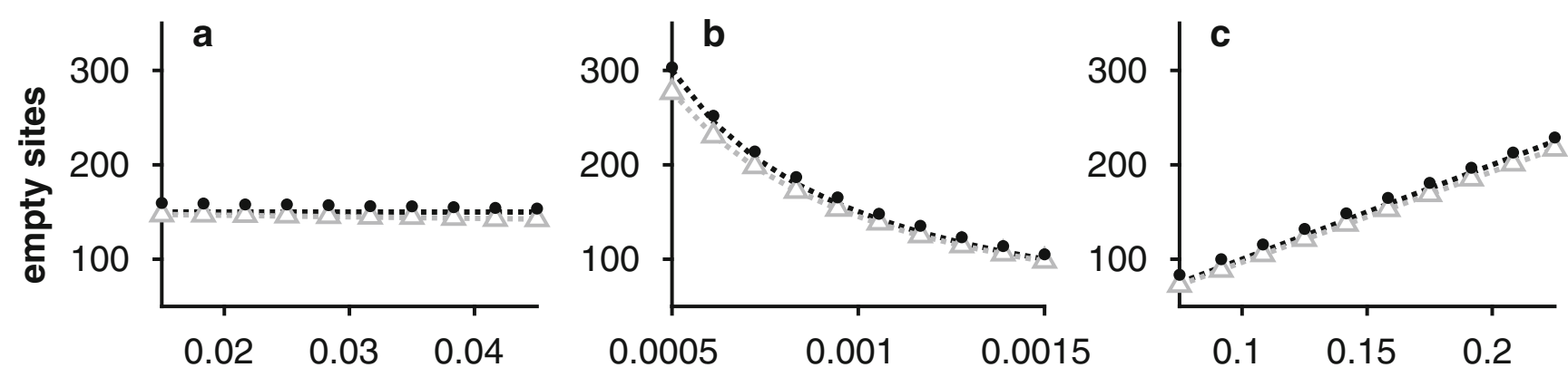

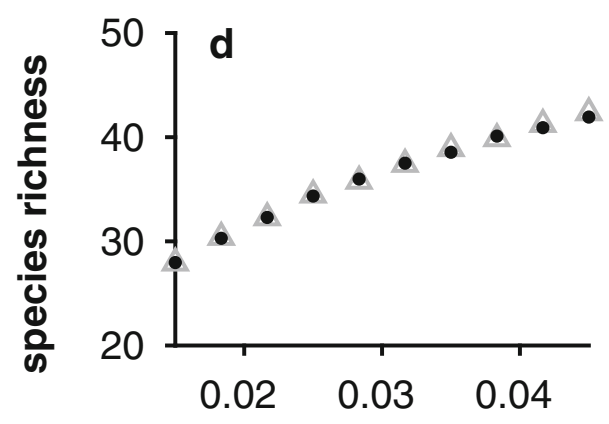

immigration rate

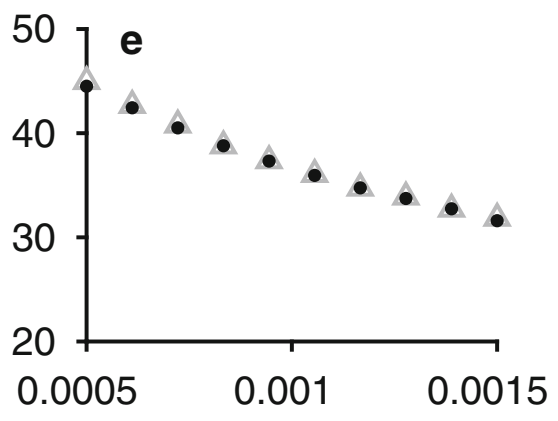

reproduction rate

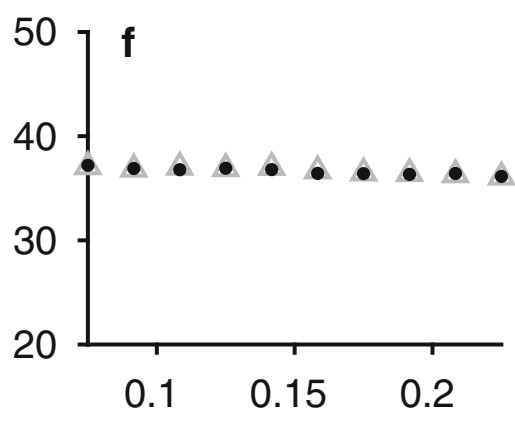

mortality rate
Fig. 5 Parameter dependence of the model outcome. The plot shows the results of the stochastic model averaged over 1000 model runs for a growing habitat with a linear growth rate of $g=10$ year $^{-1}$ (black dots) and a static habitat with a habitat capacity of $K_{0}=1000$ (grey triangles). a-c Mean number of empty sites and $\mathbf{d}-\mathbf{f}$ mean species richness, after a transient of 100 years for varying values of immigration rate $m$, reproduction rate $r$, and mortality rate $d$ (non-varied parameters are taken as in Fig. 4). Additionally shown is the equilibrium number $E^{*}$ of empty sites from the ODE model (dotted lines) for the growing (black) and static habitat (grey) 
year. In the later stages of habitat development (after more than 100 years), colonization and extinction rates are almost equal, with the colonization rate being slightly higher, which results in the very slow increase of species richness.

Even though species richness increases as a smooth continuous function of time, it exhibits in fact two different growth phases, which become apparent as straight lines in a double-logarithmic plot with different slopes for small and large times (Fig. 6 inset). Thus, the increase of species richness can be approximated as a power law $S(t) \sim t^{\alpha}$ with an initial, accelerating super-linear scaling of $\alpha \approx 1.3$, followed by slow sub-linear growth with a scaling exponent of $\alpha \approx$ 0.13 . These two scaling regimes intersect at a simulation time of about 30 years. This transition time roughly coincides with the time point when $E(t)$ undergoes its maximum, and it separates the two different growth phases. Thus, community development is characterized by two phases: The first phase corresponds to a rapid early buildup of empty sites, a fast increase of species richness, and a slow increase in total species abundance. The second phase corresponds to a steady state of empty sites, a slow increase of species richness, and a linear increase in the total number of individuals. As shown in Fig. 11 in Appendix 3, very similar patterns arise for a slowgrowing habitat without overshooting. Although in this case there is no overshooting in the number of empty sites, the other characteristics (e.g., two scaling regimes, temporal overshooting of colonization and extinction rates, etc.) remain unaffected.

On top of this overall temporal development, community dynamics in the stochastic model exhibit the typical hallmarks known from neutral theory (Hubbell 2001; Rosindell et al. 2011). This is investigated in Fig. 7, which shows the dependence of local community dynamics on the relative species abundance in the metacommunity.
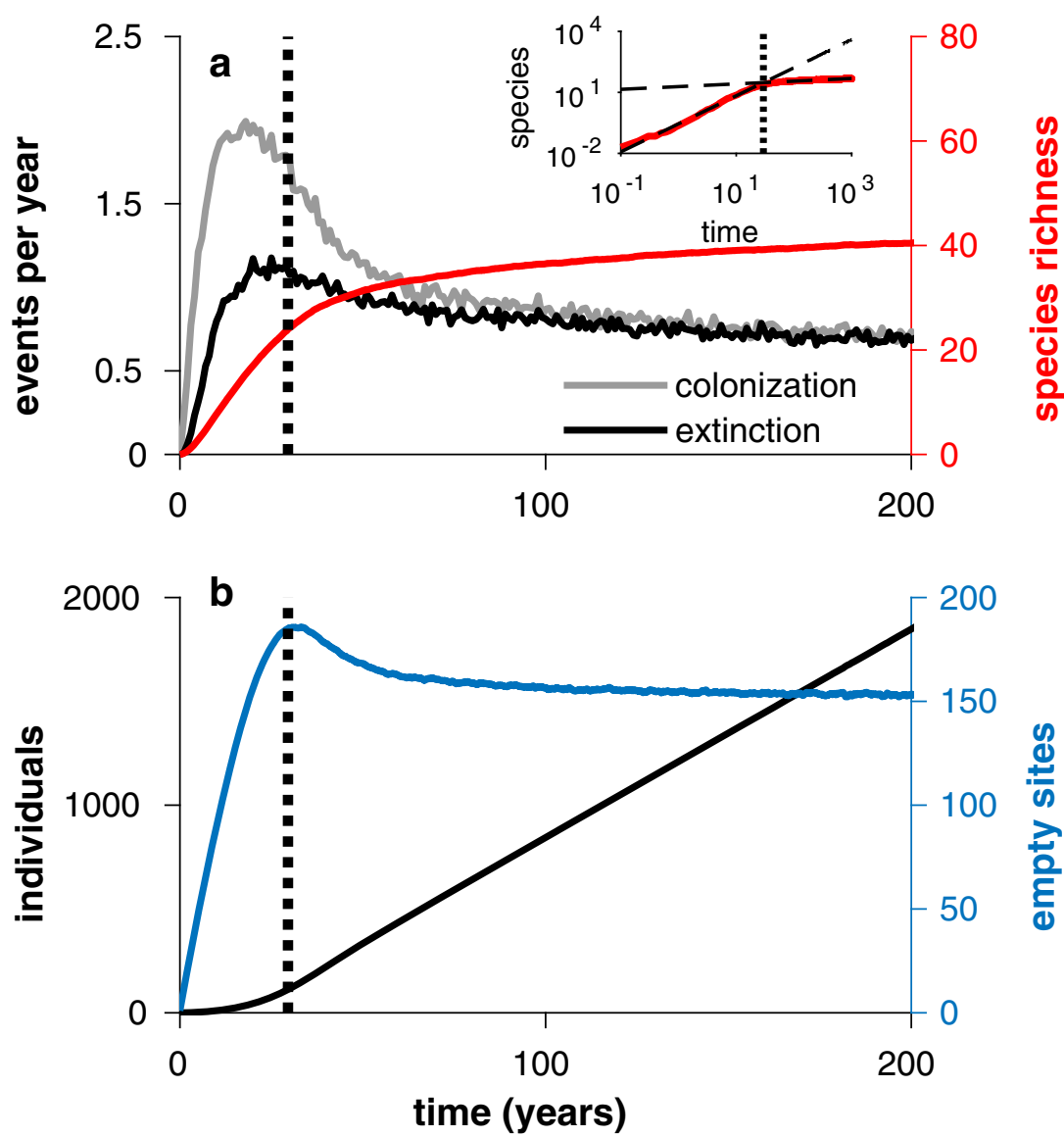

Fig. 6 Typical simulation outcome in a case with overshooting of empty sites $(\mathrm{g} / \mathrm{m}>d / \mathrm{r})$. Shown are the results of the stochastic model, averaged over 1000 simulation runs for a duration of 200 years: a mean species richness (red), colonization rate (grey), and extinction rate (black) and $\mathbf{b}$ mean number of individuals (black) and empty sites (blue). The inset in a shows the long-term development of the mean species richness (red) over 1000 years on a double-logarithmic scale, revealing two scaling regimes over either shorter or longer time. Also shown are linear fits (black dashed lines) for each scaling regime with fitted slope of $\alpha_{1}=1.3$ in the first regime and $\alpha_{2}=0.13$ in the second regime. The transition between the two scaling regimes is obtained by the intersection of the two fitting lines, yielding a transition time of 30 years (shown as vertical dotted lines). This transition time corresponds to the maximum value of $E(t)$ in $\mathbf{b}$. Parameter values as in Fig. 4. The corresponding results of a parameterization without overshooting are shown in Fig. 11 in Appendix 3 
The figure reveals that the stochastic model is characterized by stochastic drift and repeated extinction and colonization events. In particular, the average time a species $i$ is present in the habitat increases with its relative abundance in the metacommunity, $N_{\text {meta, } i}$ (Fig. 7a), its average time of first establishment decreases with $N_{\text {meta, } i}$ (Fig. 7b), its average number of extinction events follows a unimodal function of $N_{\text {meta, } i}$ (Fig. $7 \mathrm{c}$ ), and in general, its relative abundance in the simulated habitat is strongly correlated to its relative abundance in the metacommunity. Compared with a non-growing community, the dynamics of a growing community is characterized by stronger turnover, a reduced time of being present in the community, an elevated time of first establishment, and a drastically increased number of extinction events, in particular for more abundant species in the metacommunity (Fig. 7).
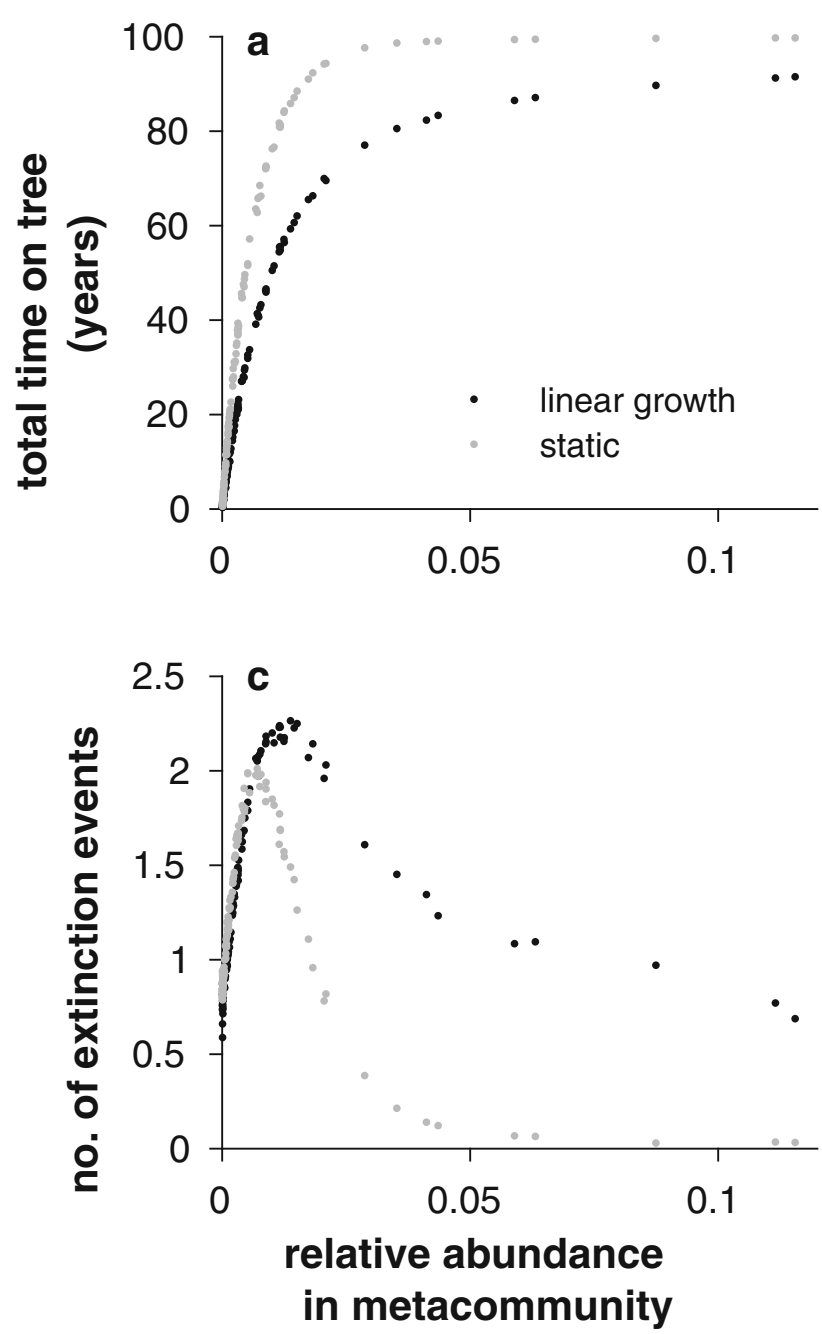

Fig. 7 Dependence of local community dynamics on relative species abundance in the metacommunity. a Average total time a species is present in the habitat, $\mathbf{b}$ average time of first establishment, and $\mathbf{c}$ average number of extinction events as a function of the relative abundance of a species in the metacommunity. d Correlation of the
Finally, in Fig. 8, we compare the results of our stochastic model with field data of epiphyte abundances and species richness per tree collected in a lowland forest in Panama (Zotz and Schultz 2008) - the same data that we used to parameterize the external species pool (Fig. 9 in Appendix 1). The data set also contains information about the diameter of trees, which can be used as a proxy for tree age. This allows us to obtain a dynamic picture of the colonization process by plotting the empirically observed values of epiphyte abundance and species richness on different trees as a function of tree diameter (Fig. 8a, b). Epiphyte abundance grows about exponentially with tree diameter. In contrast, the growth of epiphyte species richness is more moderate, being nearly linear over the whole range. With increasing tree diameter, the data become more and more sparse, resulting in large error bars. Despite this uncertainty about the form of tree growth for
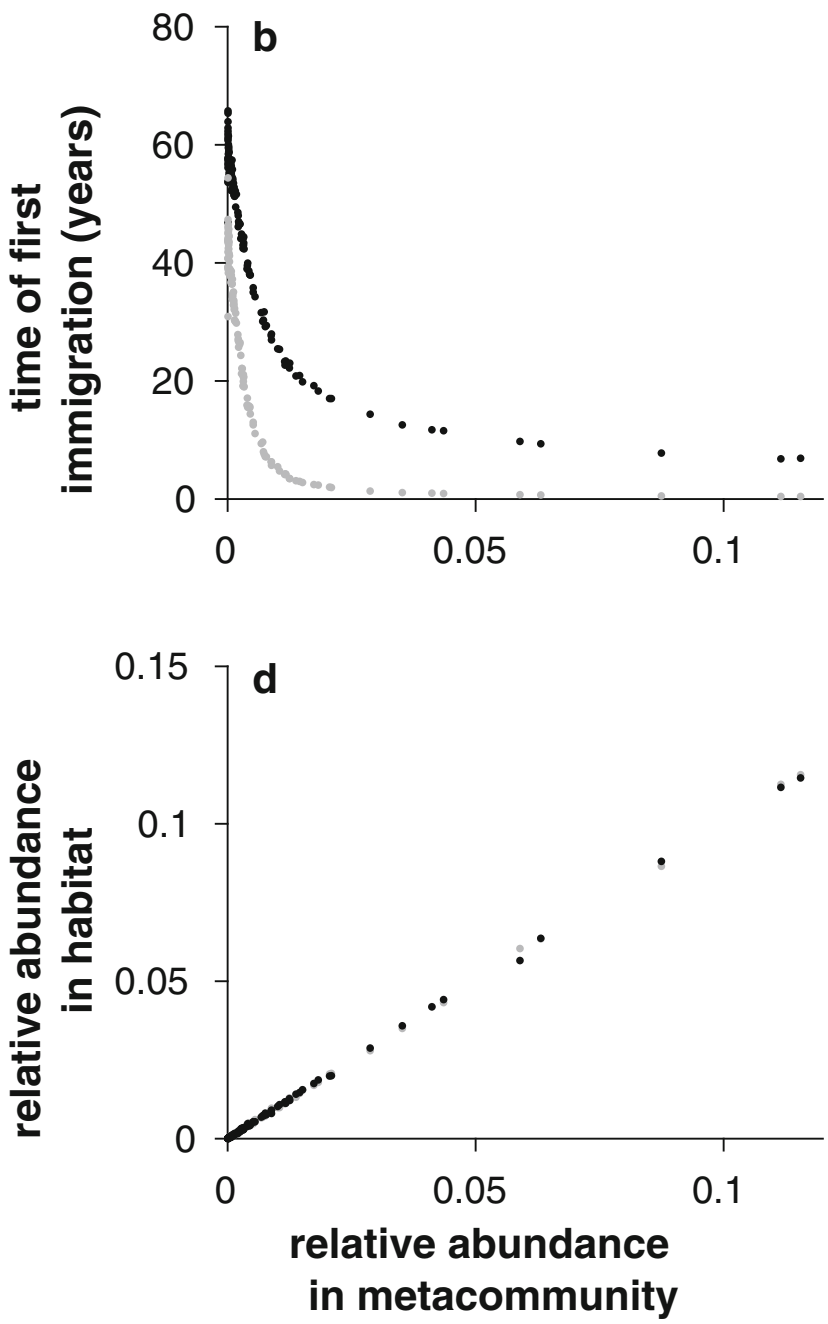

relative abundance of a species in the simulated habitat and its relative abundance in the metacommunity. Depicted are mean values of the stochastic model over 1000 model runs for a simulation time of 100 years, for a linear growing habitat (black) and a static habitat (grey) 
large diameters, the abundance and species richness of epiphytes on a tree are clearly related: Trees with a larger number of epiphyte individuals tend to have larger epiphyte species richness (Fig. 8c).

A very similar relationship of epiphyte abundance and species richness is obtained by the stochastic model (Fig. 8c). To obtain a good fit to the empirical data, we slightly modified our standard literature-based model parameters (see "Parameterization and data" section), reducing the reproduction rate to $r=0.0007$ year $^{-1}$ and elevating the immigration rate to $m=0.04$ year $^{-1}$ - a parameter regime with minor overshooting of empty sites $(g / m=1.16 d / r)$. As shown in Fig. $8 \mathrm{c}$, the model is able to reproduce the empirical abundance-diversity relationship from the epiphyte census. This figure, however, does not give insights into the temporal development during the colonization process.

To be able to compare the model results, which describe the state of the epiphyte community as a function of time, with the
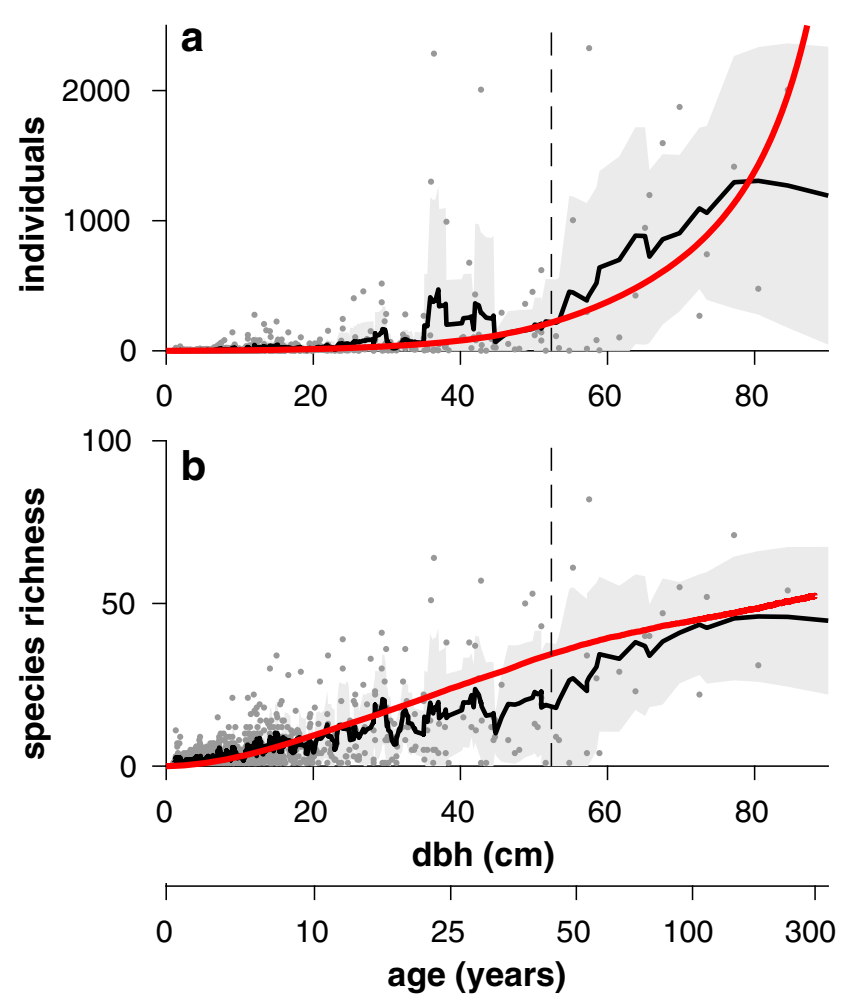

Fig. 8 Comparison of model results against empirical data on vascular epiphytes in a lowland forest (Zotz and Schultz 2008, see "Parameterization and data" section). a, b Development of epiphyte abundances (a) and species richness (b) as a function of tree diameter (dbh) and estimated tree age (see below). Empirical data on a tree are shown as grey dots (standard deviation indicated by grey-shaded area). The black lines show a running moving average through the data with a window length of $10 \mathrm{~mm}$. The red lines show the corresponding model results averaged over 100 model runs for $r=0.0007$ year $^{-1}$ and $m=$ 0.04 year $^{-1}$ (other model parameters as in Fig. 4). c The number of epiphyte species on a tree in relation to the number of individuals in the empirical data (black dots) and simulated with the model (red line). $\mathbf{d}$ The empirical data, which contain information about the tree diameter, the relationship between tree age and tree diameter, i.e., the tree growth curve, needs to be known. To estimate this relationship, we identified common values of the number of epiphyte individuals in the numerical simulation and in the census data and plotted the associated values of tree age in the simulation and tree diameter ( $\mathrm{dbh}$ ) in the field against each other in a single plot (black dots in Fig. 8d). In the same way, we also plotted the associated values of modeled tree age and measured tree diameter for common values of epiphyte species richness (grey triangles in Fig. 8d). Both cases lead to a similar saturating tree growth curve (Bowmen et al. 2013) that can be fitted with a Monod function, $d b h(t)=\frac{99.8 t}{39.5+t}$ (blue line in Fig. 8d), and subsequently can be used to convert tree age from the model to tree diameter. Having performed this calibration, we are able to compare the community dynamics from the model with the field data. As shown in Fig. 8a, b, the simulated time course of both epiphyte abundance and
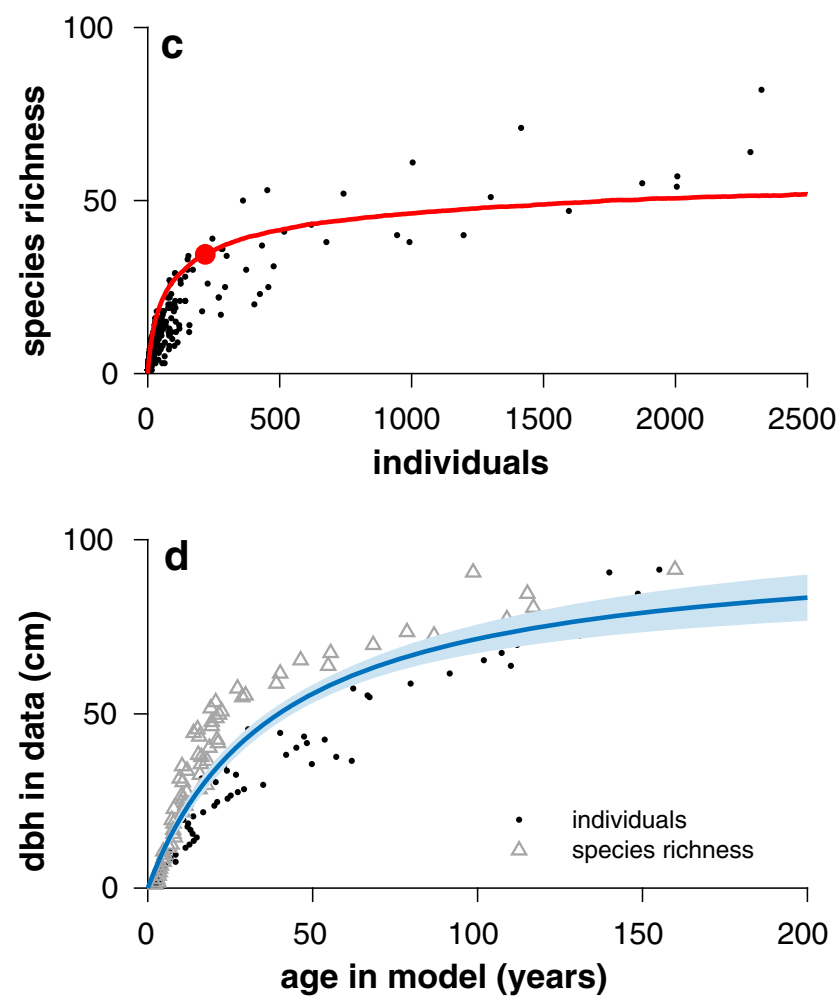

relationship of tree age, $t$, in the model and tree diameter, $d b h$, in the census, obtained by relating the time stamps, or diameter values, of common values of the either simulated, or empirically obtained, number of individuals (black dots) and species richness (grey triangles). The blue line shows a least squares fit $\left(r^{2}=0.85\right)$; blue-shaded area indicates 0.95 confidence bounds) through the empirical data by a Monod function, $d b h(t)=d_{\max } \frac{t}{t_{M}+t}$, with $d_{\max }=99.8 \pm 9.3 \mathrm{~cm}$ and $t_{M}=39.5 \pm 8.1$ year. This fitting function was used to relate tree diameter and age in $\mathbf{a}$ and $\mathbf{b}$. Vertical dashed lines in $\mathbf{a}$ and $\mathbf{b}$ and the red circle in $\mathbf{c}$ indicate the state with maximal number of empty sites in the simulation 
species richness falls well within the empirical error bars, indicating that our stochastic model is able to capture the development of an epiphyte community on a growing tree in accord to empirical observations. Even though the number of empty growing sites was not directly observed in the census, the transition between the two characteristic phases of community development, separated by the intermittent maximal number of empty sites in the model, is still apparent in the data by the reduced increase of species richness and the associated change in curvature in Fig. 8b, c.

\section{Discussion}

Fifty years after its original publication, MacArthur and Wilson's (1967) book on the Theory of Island Biogeography remains one of the most influential publications in ecology (Warren et al. 2015; Patiño et al. 2017). This equilibrium theory did not consider changes in island size. Although a later extension of the original theory, i.e., the general dynamic theory of oceanic island biogeography (Whittaker et al. 2008), accommodated changes in island size over geological time scales, ours seems to be the first attempt to study the expected changes in species numbers and abundance of organisms on habitat islands that change in size over ecological timescales. To analyze the effect of a continuously changing habitat size on community assembly and species richness patterns, we developed a model based on Hubbell's (2001) neutral theory. In our model, we neglect speciation processes (i.e., we assume that diversity is maintained by immigration from the metacommunity and not by speciation) and the zero-sum assumption made in the original theory and assume linear growth of the habitat capacity.

There are a several noteworthy results. For one, we found that in spite of pronounced initial differences in the dynamics, the species richness of a linearly growing and a static habitat of the same size converge (Fig. 5). In a static habitat, the species richness reaches an equilibrium rather early, whereas in the dynamic habitat, the species richness continuously increases as more growing sites become available.

As a second remarkable result, we found that in a growing habitat, the number of empty sites reaches an equilibrium value while habitat size and, consequently, the number of individuals continue to increase. This implies that the habitat capacity is never fully utilized by the community of dependent organisms: There is always unoccupied space. Furthermore, this equilibrium value $\left(E^{*}=d / r\right.$ for linear habitat growth) is independent of the habitat growth rate. This means that independent of how fast the habitat is growing, the number of empty sites always approaches the same steady state. The size of the habitat growth rate, however, determines the pattern in which this equilibrium is reached: In a slowly growing habitat $(g / m<d / r)$, the number of empty sites monotonically increases and saturates towards equilibrium, whereas in a rapidly growing habitat $(g / m>d / r)$, the number of empty sites temporarily exceeds the equilibrium level going through an intermediate maximum.

Thirdly, we were able to separate two characteristic phases of community development. In the initial phase, the number of empty sites has not reached its equilibrium value and there is a fast increase in species richness. The fast increase in species richness corresponds to the peak of the colonization rate in this phase. The second phase is characterized by an equilibrium number of empty sites, a continuous increase in total abundance, and a very slow increase in species richness.

Our proposed model bears some similarities to the generally dynamic theory of biogeography for oceanic islands (Whittaker et al. 2007, 2008; Borregaard et al. 2015, 2017; Lim and Marshall 2017) - albeit on a very different timescale. Taking into account the ontogeny of oceanic islands that after emergence typically grow in size, shrink, and submerge within a few million years, this theory predicts the temporal change of insular diversity in geological time in relation to a changing habitat capacity. These studies demonstrate a typical temporal development of habitat capacity, species richness, colonization rates, and extinction rates during island development, with many parallels to our simulation results (Fig. 6). For example, a high colonization rate in the beginning of habitat development was also proposed by Whittaker et al. (2008) and Borregaard et al. (2017) in their theory for oceanic islands. Also similar to our equilibrium value in the number of empty sites (Eq. 7), the dynamic theory of oceanic islands predicts that species richness never reaches its habitat capacity, i.e., that empty niche space exists permanently throughout island ontogeny (Borregaard et al. 2015). Despite these similarities, one should keep in mind that compared with our model, the general dynamic theory operates on very different (evolutionary and geological) timescales. Consequently, processes such as speciation events, which are important on geological timescales, play no role and can be neglected in our model where the timescale is set by the age of a tree.

Our model results are in line with observations from longterm monitoring studies of vascular epiphytes (Fig. 8) presented here, as well as the few other studies with repeated censuses that are available in the literature: They all report consistent increases in abundance over time periods of up to a decade without signs of saturation (Schmit-Neuerburg 2002; Laube and Zotz 2006, 2007; Einzmann and Zotz 2017). Unpublished direct observations from two mature tropical forest ecosystems in Panama indicate that this trend does not change, at least for another decade (Zotz, Mendieta-Leiva and Weichgrebe, unpubl. results). A noteworthy case is the most 
recent report by Einzmann and Zotz (2017): These authors found, on average, a threefold increase in epiphyte abundance in more than 600 host trees over less than a decade. However, due to the scarcity of empirical observations, in particular with respect to the temporal development, our findings are still difficult to transfer and compare with the situation in nature. This is particularly true with respect to the number of empty sites, as it is unclear how many unused sites there actually are, a problem that is also encountered with the application of the concept of safe sites (sensu Harper 1977) in plant population biology. There is much need for long-term monitoring studies and transplant experiments to establish both patterns and mechanisms behind the low densities of epiphytes in many lowland forests (e.g., Zotz and Vollrath 2003).

Studies of epiphyte species richness in relation to tree size usually indicate a strong positive relationship (Flores-Palacios and García-Franco 2006; Laube and Zotz 2006; Zotz and Schultz 2008). However, there are also a few observations that suggest no positive relationship (Boelter et al. 2011) or even saturation of species richness with tree size (Hietz and HietzSeifert 1995). Noteworthy, the latter observation was made in a montane setting. Wet montane forests are mostly much more epiphyte-rich than the typical lowland forest (Gentry and Dodson 1987; Zotz 2016), which also suggests different community dynamics. There are a few studies to test this notion directly (but see Acuña-Tarazona et al. 2015). The strong increase in species richness with tree size found in most studies corresponds to the development in the first phase of the model simulation. In the simulation, the increase of species richness slows down when the number of empty sites has reached equilibrium as the immigration of individuals, which only depends on the number of empty sites, becomes less influential compared with reproduction. In addition, with time, the most abundant species of the metacommunity are already present in the habitat, and immigration of new species becomes less and less likely.

This development of species richness with tree size simulated with the model is similar to the positive-asymptotic relationship proposed by Flores-Palacios and García-Franco (2006). These authors proposed a scenario with no further increase in species richness on very old trees, which have reached their maximum height, and possibly a decrease in species richness on decaying trees. However, they did not find evidence for this scenario in field data. In contrast to their suggestion, we found a slow increase in species richness even on very big trees, in the model as well as the data that was available to us (Fig. 8b). The model also suggests an ongoing increase of epiphyte abundance for large trees, going beyond 2000 individuals for the biggest trees (Fig. 8a). Taken together, there is no evidence that growing trees reach a maximum number of species. Depending on the parameter values, the time until the number of empty sites on a tree reaches their equilibrium can vary substantially. Especially the growth curve of the tree can influence the length of this transient phase. The assumption of a linear growth curve is a very strong simplification because it neglects the well-known increase of structural complexity with tree age and tree size. That is arguably the reason why the typical lag phase of epiphyte community development (Taylor and Burns 2015) is not captured by the current model, but could easily be integrated into future model versions by considering more realistic growth curves $K(t)$.

There is a considerable body of literature on epiphyte community structure and dynamics (reviewed by Mendieta-Leiva and Zotz 2015), but as already noted by Kitching (2006), there has been a lack of theoretical frameworks to guide this research. This resulted in a high level of idiosyncrasy in individual studies, which leads to problems with integration and synthesis. With a number of recent publications, this situation has started to change. For example, Burns and co-workers (Burns 2007; Blick and Burns 2009; Burns and Zotz 2010) have introduced a network and a metacommunity approach to the analysis of epiphyte communities, Taylor and Burns (2015) proposed an island ontogeny framework for the analysis of epiphyte community dynamics, and Woods (2017) introduced a species accumulation model. Ours is the most recent attempt to provide theory for the study of the community dynamics of organisms in a habitat that itself is alive and growing. Probably the most remarkable finding is the expectation that - after an initial transient phase - there is a constant number of empty sites.

Being a conceptional study, our developed model is limited by many simplifications that can be addressed in subsequent work. We see four main avenues for future model extensions: First, it would be important to generalize the monotonously increasing habitat growth curves to more complex function, allowing for phases of decreasing habitat size (e.g., due to reduced number of growing sites in aging trees) (Fox et al. 2001). Second, the current model lacks an explicit consideration of the spatial structure within a tree, that is, it assumes population dynamics to be equal for all individuals irrespective of their spatial position. This is unrealistic as environmental factors such as light, water, and nutrient inputs, as well as branch (or twig) diameters, will differ with the location within the canopy, leading to height-specific rates of mortality (Zotz 2007). Furthermore, species interactions will depend on spatial distances (e.g., local reproduction by individual species are more likely in empty sites next to sites that these species currently occupy) and will be spatially correlated (e.g., mortality will be spatially concentrated when whole branches break) (Cabral et al. 2015). Taking such spatially explicit processes into account potentially could increase the variance of the trends observed in the current simulations. Third, the 
neutral hypothesis most likely will be violated in real systems because ecophysiological and morphological differences among epiphyte species play an important role to determine their success in different parts of a tree (Petter et al. 2016). For example, shaded branches in general will support different species than exposed tips. These factors might dynamically be related to the age of a tree. Thus, changes in microsite conditions as a tree grows could drive much higher rates of local mortality, affecting species very differently. Such increasing microsite diversity through time could be a decisive factor contributing to the observed increase in epiphyte species richness. Finally, one interesting perspective for future model studies would be to scale up from the epiphyte community on an individual, growing tree to the epiphyte communities in a stand of growing trees, which in turn will develop in or out of synchrony.

\section{Appendix 1}

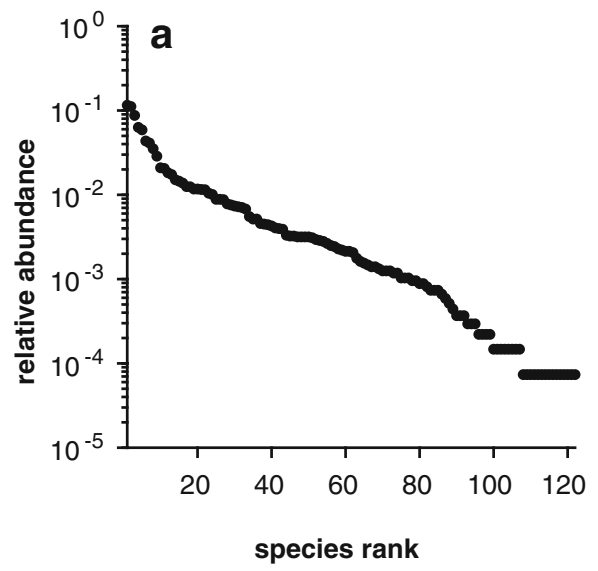

Fig. 9 Characteristics of the metacommunity. As external species pool in the simulations, we used data on vascular epiphytes collected in a census from a 0.4-ha plot in a lowland forest in Panama (Zotz and Schultz 2008).
Author contributions BB and GZ conceived the study; LS and JH implemented the model; LS and BB prepared the manuscript. All authors critically revised the manuscript.

Funding information LS was funded by the Ministry of Science and Culture of Lower Saxony (Niedersächsiches Ministerium für Wissenschaft und Kultur (MWK)) within the research training program IBR (Interdisciplinary Approach to Functional Biodiversity Research). Funding for field work in Panama was provided by Deutsche Forschungsgemeinschaft (DFG ZO 94/5-1).

\section{Compliance with ethical standards}

Conflict of interest The authors declare that they have no conflict of interest.

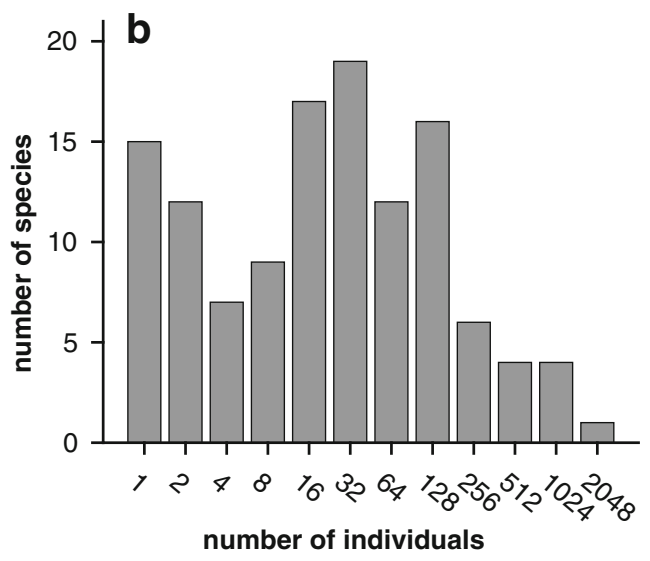

The data set comprises a total of 13,574 epiphyte individuals from 122 species. Shown are $\mathbf{a}$ the corresponding rank abundance curve and $\mathbf{b}$ the frequency histogram of species abundances (Preston plot) 


\section{Appendix 2}

Fig. 10 Dependence on the functional form of habitat growth. The plot shows the time dependence of the habitat capacity $K(t)$ (black), the

simulated number of empty sites $E(t)$ (blue), and the total number of individuals $N(t)$ (red) over 150 years for different forms of habitat growth: a linear growth $K(t)=10 t$ (Eq. 1), b exponential growth $K(t)=\exp (0.053 t)$, and $\mathbf{c}$ logarithmic growth $K(t)=100$ $\log (t+1)$. In all cases, $E(t)$ reaches a stationary state, despite the different (linear, accelerating, or declining) changes in habitat growth
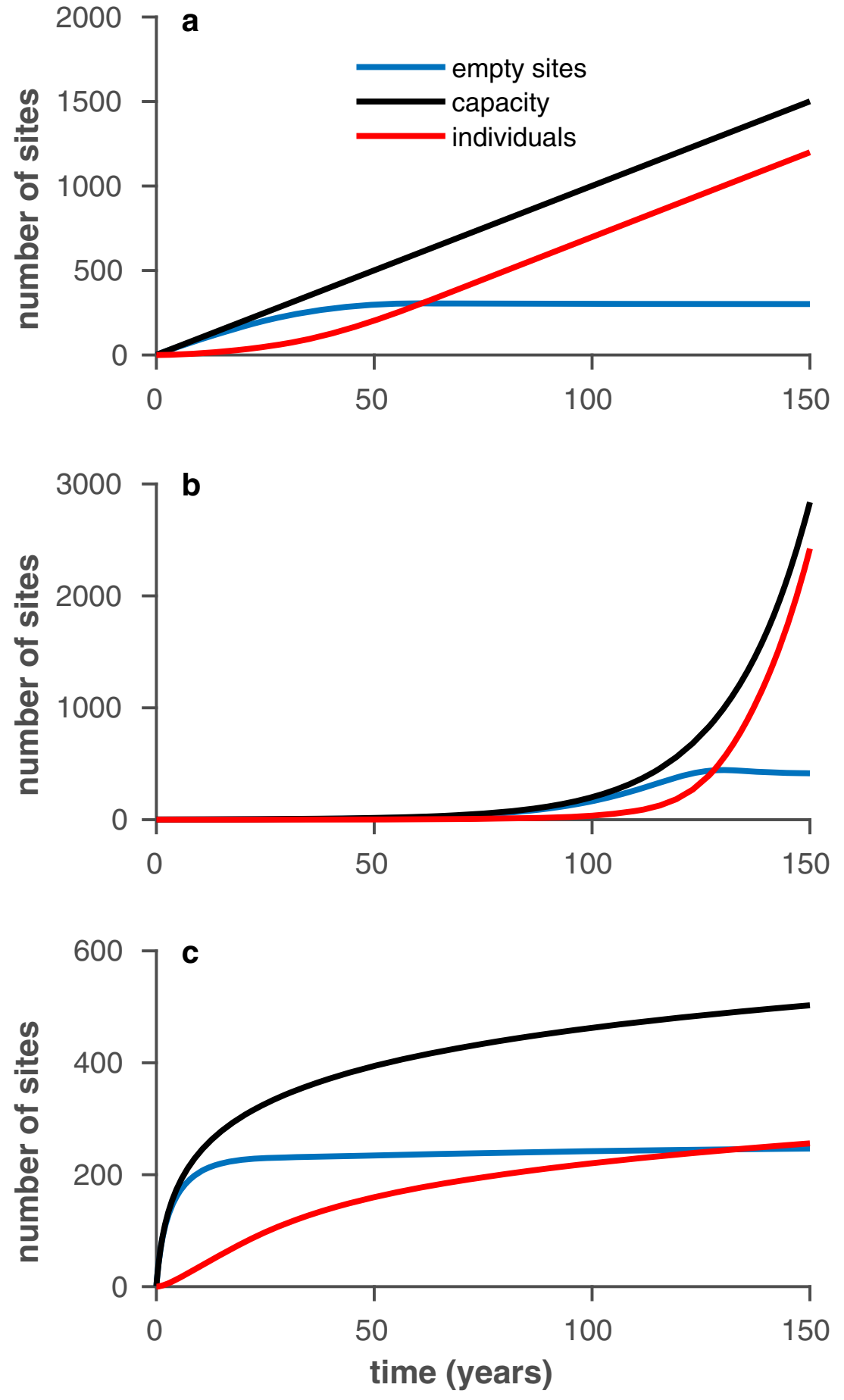


\section{Appendix 3}
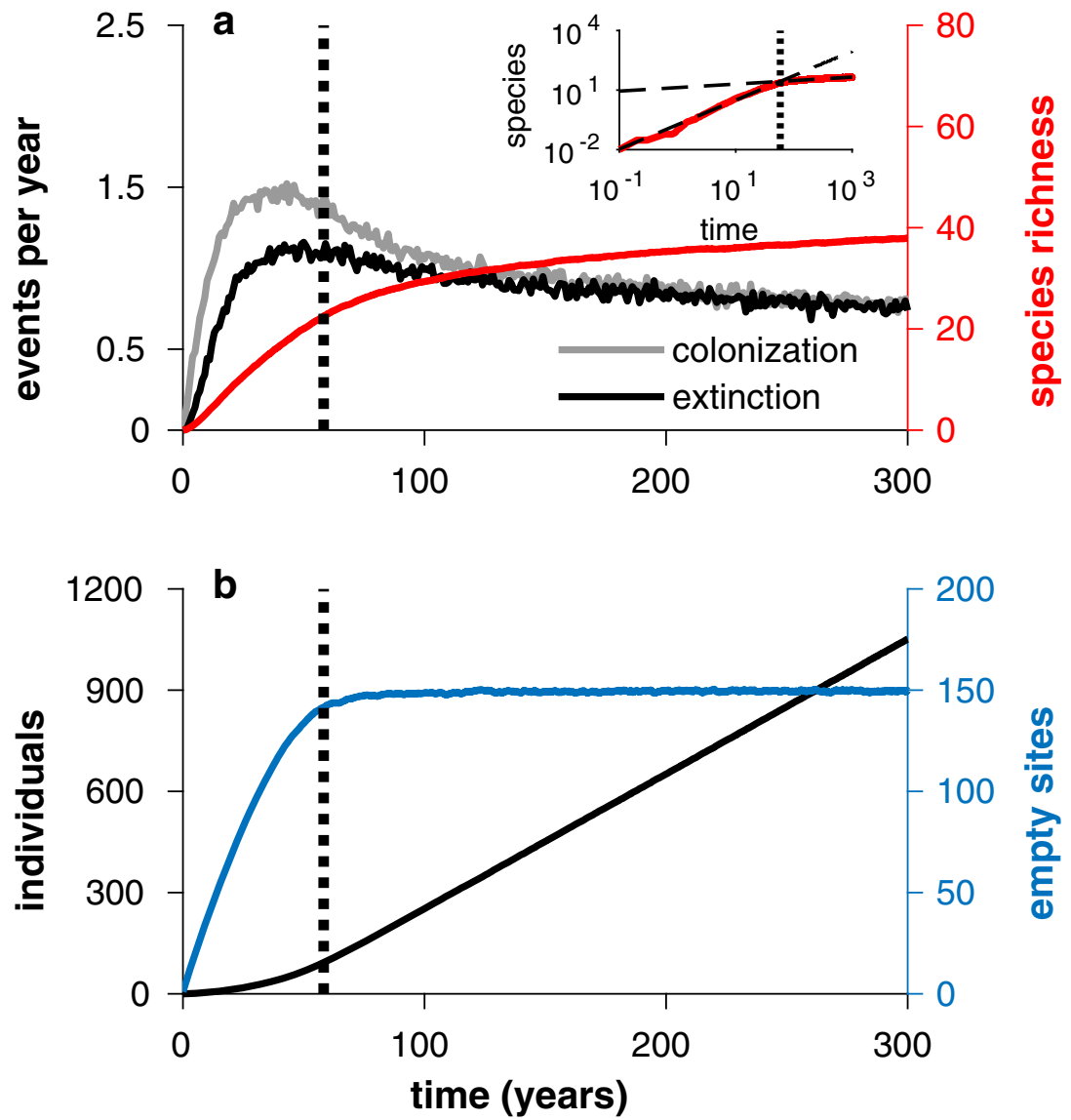

Fig. 11 Typical simulation outcome in a case without overshooting of empty sites $(g / m<d / r)$ (compare with Fig. 2). Shown are the results of the stochastic model, averaged over 1000 simulation runs for a duration of 300 years: a mean species richness (red), colonization rate (grey), and extinction rate (black) and $\mathbf{b}$ mean number of individuals (black) and empty sites (blue). The inset in a shows the long-term development of the mean species richness (red) over 1000 years on a double-logarithmic scale, revealing two scaling regimes for either small or large time. Further

\section{References}

Acuña-Tarazona M, Toledo-Aceves T, Flores-Palacios A, Sosa VJ, Martínez ML (2015) Post-stripping recolonization of vascular epiphytes in cloud-forest fragments in Mexico. J Trop Ecol 31:499508. https://doi.org/10.1017/S0266467415000395

Blick R, Burns K (2009) Network properties of aboreal plants: are epiphytes, mistletoes and lianas structured similarly? Perspect Plant Ecol Syst 11:41-52. https://doi.org/10.1016/j.ppees.2008.10.002

Boelter, C.R., Zartman, C.E., Fonseca, C.R., 2011. Exotic tree monocultures play a limited role in the conservation of Atlantic Forest epiphytes. Biodivers. Conserv. 20, 1255-1272

Borregaard MK, Matthews TJ, Whittaker RJ (2015) The general dynamic model: towards a unified theory of island biogeography? Glob Ecol Biogeogr 25:805-816. https://doi.org/10.1111/geb.12348

Borregaard MK, Amorim IR, Borges PAV, Cabral JS, Fernández-Palacios JM, Field R, Heaney LR, Kreft H, Matthews TJ, Olesen JM, Price J, Rigal F, Steinbauer MJ, Triantis KA, Valente L, Weigelt P, Whittaker RJ (2017) Oceanic island biogeography through the lens of the shown are linear fits (black dashed lines) for each scaling regime with fitted slope of $\alpha_{1}=1.2$ in the first regime and $\alpha_{2}=0.17$ in the second regime, respectively. The transition between the two scaling regimes is obtained by the intersection of the two fitting lines, yielding a transition time of 58 years (shown as vertical dotted lines). This transition time roughly corresponds to the time when $E(t)$ reaches saturation in $\mathbf{b}$. Parameterization without overshooting $\left(g=4\right.$ year $\left.^{-1}\right)$, other parameters as in Fig. 6

general dynamic model: assessment and prospect. Biol Rev 92: 830-853. https://doi.org/10.1111/brv.12256

Bowmen DMJS, Brienen RJW, Gloor E, Phillips OL, Prior LD (2013) Detecting trends in tree growth: not so simple. Trends Plant Sci 18: 11-17. https://doi.org/10.1016/j.tplants.2012.08.005

Burns KC (2007) Network properties of an epiphyte metacommunity. J Ecol 95:1142-1151. https://doi.org/10.1111/j.1365-2745.2007.01267.x

Burns KC, Zotz G (2010) A hierarchical framework for investigating epiphyte assemblages: networks, meta-communities, and scale. Ecology 91:377-385. https://doi.org/10.1890/08-2004.1

Cabral JS, Petter G, Mendieta Leiva G, Wagner K, Zotz G, Kreft H (2015) Branchfall as a demographic filter for epiphyte communities: lessons from forest floor-based sampling. PLoS One 10:e0128019. https://doi. org/10.1371/journal.pone.0128019

Chave J (2004) Neutral theory and community ecology. Ecol Lett 7:241253. https://doi.org/10.1111/j.1461-0248.2003.00566.x

Condit R, Pitman N, Leigh EG, Chave J, Terborgh J, Foster RB, Núñez P, Aguilar S, Valencia R, Villa G, Muller-Landau HC, Losos E, Hubbell SP (2002) Beta-diversity in tropical forest trees. Science 295:666-669. https://doi.org/10.1126/science.1066854 
Einzmann HJR, Zotz G (2017) "No signs of saturation": long-term dynamics of vascular epiphyte communities in a human-modified landscape. Biodivers Conserv 26:1393-1410. https://doi.org/10.1007/s10531017-1306-Z

Flores-Palacios A, García-Franco JG (2006) The relationship between tree size and epiphyte species richness: testing four different hypotheses. J Biogeogr 33:323-330. https://doi.org/10.1111/j.1365-2699.2005.01382.x

Fox JC, Ades PK, Bi H (2001) Stochastic structure and individual-tree growth models. For Ecol Manag 154:261-276. https://doi.org/10. 1016/S0378-1127(00)00632-0

Gentry AH, Dodson CH (1987) Diversity and biogeography of neotropical vascular epiphytes. Ann Mo Bot Gard 74:205-233. https://doi. org/10.2307/2399395

Harper JL (1977) Population biology of plants. London Academic Press, London

Hietz P (1997) Population dynamics of epiphytes in a Mexican humid montane forest. J Ecol 85:767-775. https://doi.org/10.2307/ 2960600

Hietz P, Hietz-Seifert U (1995) Composition and ecology of vascular epiphyte communities along an altitudinal gradient in central Veracruz, Mexico. J Veg Sci 6:487-498. https://doi.org/10.2307/3236347

Honerkamp J (1993) Stochastic dynamical systems: concepts, numerical methods, data analysis. John Wiley \& Sons, New York

Hubbell SP (2001) The unified neutral theory of biodiversity and biogeography. Princeton University Press, Princeton

Kelly DL, Tanner EVJ, Lughadha EMN, Kapos V (1994) Floristics and biogeography of a rain forest in the Venezuelan Andes. J Biogeogr 21:421. https://doi.org/10.2307/2845760

Kier G, Kreft H, Lee TM, Jetz W, Ibisch PL, Nowicki C, Mutke J, Barthlott W (2009) A global assessment of endemism and species richness across island and mainland regions. Proc Natl Acad Sci 106:9322-9327. https://doi.org/10.1073/pnas.0810306106

Kitching RL (2006) Crafting the pieces of the diversity jigsaw puzzle. Science 313:1055-1057. https://doi.org/10.1126/science.1128447

Kreft H, Jetz W, Mutke J, Kier G, Barthlott W (2007) Global diversity of island floras from a macroecological perspective. Ecol Lett 11:116 127. https://doi.org/10.1111/j.1461-0248.2007.01129.x

Laube S, Zotz G (2006) Long-term changes of the vascular epiphyte assemblage on the palm Socratea exorrhiza in a lowland forest in Panama. J Veg Sci 17:307-314. https://doi.org/10.1111/j.1654-1103.2006.tb02450.x

Laube S, Zotz G (2007) A metapopulation approach to the analysis of long-term changes in the epiphyte vegetation on the host tree Annona glabra. J Veg Sci 18:613-624. https://doi.org/10.1111/j. 1654-1103.2007.tb02575.x

Lim JY, Marshall CR (2017) The true tempo of evolutionary radiation and decline revealed on the Hawaiian archipelago. Nature 543:710-713. https://doi.org/10.1038/nature21675

Lomolino MV (2000) Ecology's most general, yet protean pattern: the species-area relationship. J Biogeogr 27:17-26. https://doi.org/10. 1046/j.1365-2699.2000.00377.x

MacArthur RH, Wilson EO (1967) The theory of island biogeography. Princeton University Press, Princeton

Mendieta-Leiva G, Zotz G (2015) A conceptual framework for the analysis of vascular epiphyte assemblages. Perspect Plant Ecol Syst 17: 510-521. https://doi.org/10.1016/j.ppees.2015.09.003

Mondragón D, Valverde T, Hernández-Apolinar M (2015) Population ecology of epiphytic angiosperms: a review. Trop Ecol 56:01-39. https://doi.org/10.13140/2.1.4043.5849

Patiño J, Whittaker RJ, Borges PAV, Fernández-Palacios JM, Ah-Peng C, Araújo MB, Ávila SP, Cardoso P, Cornuault J, de Boer EJ, de Nascimento L, Gil A, González-Castro A, Gruner DS, Heleno R, Hortal J, Illera JC, Kaiser-Bunbury CN, Matthews TJ, Papadopoulou A, Pettorelli N, Price JP, Santos AMC, Steinbauer MJ, Triantis KA, Valente L, Vargas P, Weigelt P, Emerson BC (2017) A roadmap for island biology: 50 fundamental questions after 50 years of The Theory of Island Biogeography. J Biogeogr 44:963-983. https://doi.org/10.1111/jbi.12986
Petter G, Wagner K, Wanek W, Sánchez Delgado EJ, Zotz G, Cabral JS, Kreft H (2016) Functional leaf traits of vascular epiphytes: vertical trends within the forest, intra- and interspecific trait variability, and taxonomic signals. Funct Ecol 30:188-198. https://doi.org/10.1111/ $1365-2435.12490$

Ricklefs RE, Schulter D (1993) Species diversity in ecological communities: historical and geographical perspectives. University of Chicago Press, Chicago

Rosenzweig ML (1995) Species diversity in space and time. Cambridge University Press, Cambridge

Rosindell J, Hubbell SP, Etienne RS (2011) The unified neutral theory of biodiversity and biogeography at age ten. Trends Ecol Evol 26:340-348

Schmit-Neuerburg V (2002) Dynamics of vascular epiphyte vegetation in the Venezuelan lowland rain forest of the Surumoni Crane Project. $\mathrm{PhD}$ thesis, Rheinische Friedrich-Wilhelms-Universität Bonn

Taylor A, Burns K (2015) Epiphyte community development throughout tree ontogeny: an island ontogeny framework. J Veg Sci 26:902910. https://doi.org/10.1111/jvs.12289

Thomsen MS, Altieri AH, Angelini C, Bishop MJ, Gribben PE, Lear G, He Q, Schiel DR, Silliman BR, South PM, Watson DM, Wernberg T, Zotz G (2018) Secondary foundation species enhance biodiversity. Nat Ecol Evol 2:634-639

Wagner K, Mendieta-Leiva G, Zotz G (2015) Host specificity in vascular epiphytes: a review of methodology, empirical evidence and potential mechanisms. AoB Plants 7:plu092. https://doi.org/10.1093/ aobpla/plu092

Warren BH, Simberloff D, Ricklefs RE, Aguilée R, Condamine FL, Gravel D, Morlon H, Mouquet N, Rosindell J, Casquet J, Conti E, Cornuault J, Fernández-Palacios JM, Hengl T, Norder SJ, Rijsdijk KF, Sanmartín I, Strasberg D, Triantis KA, Valente LM, Whittaker RJ, Gillespie RG, Emerson BC, Thébaud C (2015) Islands as model systems in ecology and evolution: prospects fifty years after MacArthur-Wilson. Ecol Lett 18:200-217. https://doi.org/10.1111/ele.12398

Whittaker RJ, Ladle RJ, Araújo MB, Fernández-Palacios JM, Delgado JD, Arévalo JR (2007) The island immaturity—speciation pulse model of island evolution: an alternative to the "diversity begets diversity" model. Ecography 30:321-327. https://doi.org/10.1111/ j.0906-7590.2007.04761.x

Whittaker RJ, Triantis KA, Ladle RJ (2008) A general dynamic theory of oceanic island biogeography. J Biogeogr 35:977-994. https://doi. $\operatorname{org} / 10.1111 / j .1365-2699.2008 .01892 . x$

Woods CL (2017) Primary ecological succession in vascular epiphytes: the species accumulation model. Biotropica 49:452-460. https://doi. org/10.1111/btp. 12443

Woods CL, Cardelús CL, DeWalt SJ (2015) Microhabitat associations of vascular epiphytes in a wet tropical forest canopy. J Ecol 103:421430. https://doi.org/10.1111/1365-2745.12357

Zotz G (2007) Johansson revisited: the spatial structure of epiphyte assemblages. J Veg Sci 18:123-130. https://doi.org/10.1111/j.16541103.2007.tb02522.x

Zotz G (2013) The systematic distribution of vascular epiphytes - a critical update. Bot J Linn Soc 171:453-481. https://doi.org/10.1111/boj.12010

Zotz G (2016) Plants on plants - the biology of vascular epiphytes. Springer International Publishing, Switzerland

Zotz G, Schultz S (2008) The vascular epiphytes of a lowland forest in Panama - species composition and spatial structure. Plant Ecol 195: 131-141. https://doi.org/10.1007/s11258-007-9310-0

Zotz G, Vollrath B (2003) The epiphyte vegetation on the palm Socratea exorrhiza - correlations with tree size, tree age and bryophyte cover. J Trop Ecol 19:81-90. https://doi.org/10.1017/S0266467403003092

Publisher's note Springer Nature remains neutral with regard to jurisdictional claims in published maps and institutional affiliations. 